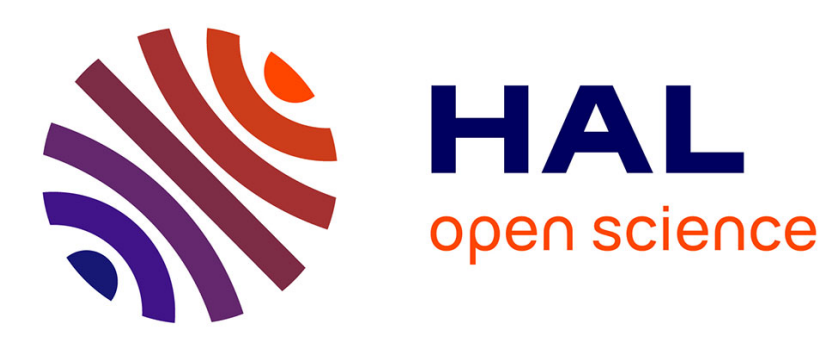

\title{
Lycopene bioavailability is associated with a combination of genetic variants
}

Patrick Borel, Charles Desmarchelier, Marion Nowicki, Romain Bott

\section{To cite this version:}

Patrick Borel, Charles Desmarchelier, Marion Nowicki, Romain Bott. Lycopene bioavailability is associated with a combination of genetic variants. Free Radical Biology and Medicine, 2015, 83, pp.238 - 244. 10.1016/j.freeradbiomed.2015.02.033 . inserm-01478367

\section{HAL Id: inserm-01478367 https://www.hal.inserm.fr/inserm-01478367}

Submitted on 20 Apr 2021

HAL is a multi-disciplinary open access archive for the deposit and dissemination of scientific research documents, whether they are published or not. The documents may come from teaching and research institutions in France or abroad, or from public or private research centers.
L'archive ouverte pluridisciplinaire $\mathbf{H A L}$, est destinée au dépôt et à la diffusion de documents scientifiques de niveau recherche, publiés ou non, émanant des établissements d'enseignement et de recherche français ou étrangers, des laboratoires publics ou privés. 


\section{Lycopene bioavailability is associated with a combination of genetic variants}

Patrick Borel $^{\mathrm{a}, \mathrm{b}, \mathrm{c}^{*}}$, Charles Desmarchelier ${ }^{\mathrm{a}, \mathrm{b}, \mathrm{c}^{* *}}$, Marion Nowicki $^{\mathrm{a}, \mathrm{b}, \mathrm{c}}$, Romain Bott ${ }^{\mathrm{a}, \mathrm{b}, \mathrm{c}}$

${ }^{a}$ INRA, UMR INRA1260, F-13005, Marseille, France (PB, CD, MN, RB)

${ }^{\mathrm{b}}$ INSERM, UMR_S 1062, F-13005, Marseille, France (PB, CD, MN, RB)

${ }^{c}$ Aix-Marseille Université, NORT, F-13005, Marseille, France (PB, CD, MN, RB)

* To whom correspondence and request for reprints should be addressed:

$\underline{\text { Patrick.Borel@univ-amu.fr }}$

UMR 1260 INRA/1062 INSERM/Aix-Marseille University

"Nutrition Obesity and Risk of Thrombosis"

Faculté de Médecine

27, boulevard Jean Moulin

13005 Marseille

France

Phone: +33 (0)491294111

Fax: +33 (0)4 91782101

** C. Desmarchelier is co-first author

Running head: Lycopene bioavailability

1 Abbreviations: ABCB1 (ATP-binding cassette, sub-family B (MDR/TAP), member 1);

2 AUC (area under the curve); BCO1 ( $\beta$-carotene 15,15'oxygenase-1); BCO2 ( $\beta$-carotene 
3 9,10'-oxygenase-2); CD36 (cluster determinant 36); CM (chylomicron); ELOVL2 (ELOVL

4 fatty acid elongase 2); HPLC (high performance liquid chromatography); ISX (intestine

5 specific homeobox); L-FABP (liver-fatty acid binding protein); LYC (lycopene); MTTP

6 (microsomal triglyceride transfer protein); PLS (partial least squares); SCARB1 (scavenger

7 receptor class $\mathrm{B}$, member 1 ); SNPs (single nucleotide polymorphisms); SOD2 (superoxide 8 dismutase 2, mitochondrial); SR-BI (scavenger receptor class B type I); VIP (variable 9 importance in the projection).

10

11 This clinical trial is registered at http://www.clinicaltrials.gov/ct2/. The ID number is 12 NCT02100774.

19 Footnote: chylomicron (CM), Lycopene (LYC), single nucleotide polymorphism (SNP), 20 partial least square regression (PLS) 


\section{Abstract}

22 Background: The intake of tomatoes and tomato products, which constitute the main dietary source of the red pigment lycopene (LYC), has been associated with a reduced risk of prostate cancer and cardiovascular disease, suggesting a protective role of this carotenoid. However, LYC bioavailability displays high interindividual variability. This variability may lead to varying biological effects following LYC consumption. Objectives: Based on recent results obtained with two other carotenoids, we assumed that this variability was due, at least in part, to several single nucleotide polymorphisms (SNPs) in genes involved in LYC and lipid metabolism. Thus, we aimed at identifying a combination of SNPs significantly associated 30 with the variability in LYC bioavailability.

31 Design: In a postprandial study, 33 healthy male volunteers consumed a test meal containing $100 \mathrm{~g}$ tomato puree, which provided $9.7 \mathrm{mg}$ all-trans LYC. LYC concentrations were measured in plasma chylomicrons $(\mathrm{CM})$ isolated at regular time intervals over $8 \mathrm{~h}$ postprandially. 1885 SNPs in 49 candidate genes, i.e. genes assumed to play a role in LYC bioavailability, were selected. Multivariate statistical analysis (partial least squares regression) was used to identify and validate the combination of SNPs most closely associated with postprandial CM LYC response.

Results: The postprandial CM LYC response to the meal was notably variable with a CV of 70\%. A significant $(P=0.037)$ and validated partial least squares regression model, which included 28 SNPs in 16 genes, explained $72 \%$ of the variance in the postprandial CM LYC

41 response. The postprandial CM LYC response was also positively correlated to fasting plasma 42 LYC concentrations $(\mathrm{r}=0.37, P<0.05)$.

43 Conclusions: The ability to respond to LYC is explained, at least partly, by a combination of 28 SNPs in 16 genes. Interindividual variability in bioavailability apparently affects the long 
45 term blood LYC status, which could ultimately modulate the biological response following 46 LYC supplementation.

47

48 Keywords: carotenoid; absorption; single nucleotide polymorphisms; nutrigenetic; genetic 49 polymorphisms 


\section{Introduction}

Lycopene (LYC) is the red pigment found in tomatoes and tomato products. It is the carotenoid found at the highest levels in the blood of Americans and the second one in the blood of Europeans. Its protective role against the development of prostate cancer and cardiovascular diseases has been suggested [1-6] but the mechanisms involved have yet to be fully elucidated. Indeed, although LYC's potential as an antioxidant is well characterized in vitro [7], there is evidence that its biological effects could also be mediated by metabolic products of LYC [8-11], a hypothesis supported by the results of recent molecular studies [11$14]$.

LYC digestion begins in the gastrointestinal lumen where digestive enzymes can modulate its bioaccessibility by facilitating its release from the food matrix to micelles [1517]. Micelles then carry LYC to the apical side of the enterocyte. LYC uptake is not only passive, as previously thought, [18] but its transport is also facilitated by two membrane proteins, SR-BI (scavenger receptor class B type I) [19], encoded by SCARB1, and CD36 (cluster determinant 36) [20]. Following uptake, it is possible that a portion of LYC may be metabolized. Indeed, enterocytes contain two enzymes with the potential to cleave LYC. The first one, $\mathrm{BCO} 1$ ( $\beta$-carotene 15,15 ' oxygenase-1), is a dioxygenase [21] that catalyzes the oxidative cleavage of LYC with an efficiency similar to that of $\beta$-carotene [22], and which has been associated with blood LYC status [23]. The second enzyme, BCO2 ( $\beta$-carotene-9,10'oxygenase), has been previously suggested to be the main LYC-cleaving enzyme [10]. Regardless of possible metabolism, the fraction of parent LYC remaining in the enterocyte is transported within the cell to the site where it is incorporated into chylomicrons (CM). The mechanism of this transport is not known, but proteins involved in intracellular transport of lipids have been suggested to be involved [24]. LYC-containing CM are secreted into the 
75 lymph, and then enter the bloodstream. LYC is assumed to stay within these lipoproteins and

76 to follow the fate of CM which is ultimately taken up by the hepatocytes [25].

77 Clinical trials dedicated to the study of LYC bioavailability have reported high 78 interindividual variability in blood and tissue LYC concentration in response to LYC intake $79[18,26-30]$. Genetic variations between individuals may provide a partial explanation of this 80 phenomenon $[31,32]$. Some genetic polymorphisms have been shown to be associated with 81 the variability in fasting blood LYC concentrations [23, 30, 33-36], and yet only one study has 82 attempted to assess the role of genetic polymorphisms involved in the variability in blood 83 LYC concentration following a LYC load [30]. Only two SNPs in BCO1 have been previously 84 shown to be associated with the variability in LYC bioavailability [30]. While promising, the 85 small number of candidate genes previously investigated calls for more studies to explain the 86 variation in a phenotype that is likely affected by numerous environmental and genetic factors 87 [31]. In fact, we have recently demonstrated that the bioavailability of the carotenoid lutein is 88 a complex phenotype that is not modulated by any single gene, but by the additive effects 89 several gene SNPs [37]. Thus, the aim of this study was to identify the combination of SNPs 90 associated with the variability in LYC bioavailability. 
Thirty five healthy, nonobese, nonsmoking male subjects were recruited for the study.

Subjects presented with normal energy consumption, i.e. $\approx 2500 \mathrm{kcal} / \mathrm{d}$ and drank $\leq 2 \%$ alcohol as total energy. They had no history of chronic disease, hyperlipidemia,

97 hyperglycemia, and were not taking any medication that might affect LYC or lipid metabolism (e.g. tetrahydrolipstatin, ezetimibe, phytosterols, cholestyramine, fibrates, etc.) during the month prior to the study or during the study period. Because of the relatively large volume of blood that was drawn during the study, subjects were required to have a blood hemoglobin concentration $>1.3 \mathrm{~g} / \mathrm{L}$ as inclusion criteria. The study was approved by the

102 regional committee on human experimentation $\left(\mathrm{N}^{\circ} 2008-\mathrm{A} 01354-51\right.$, Comité de Protection des

103 Personnes Sud Méditerranée I, France). The procedures followed were in accordance with the 104 Helsinki Declaration of 1975 as revised in 1983. The objectives and requirements of the study were fully explained to all participants before beginning the study, and informed written consent was obtained from each subject. Two subjects left the study for personal reasons

107 before participating in the postprandial experiment, leaving 33 subjects whose baseline

108 characteristics are reported in Table 1. Note that the fasting plasma LYC concentration of these subjects was relatively high as compared to previous reported data [38] suggesting that these subjects regularly consumed tomatoes and tomato products.

DNA preparation and genotyping methods

An average of $25 \mu \mathrm{g}$ of DNA was isolated from a saliva sample from each subject 114 using the Oragene kit (DNA Genotek Inc., Kanata, ON, Canada) as described in detail 115 previously [39]. DNA concentration and purity were checked by spectrophotometry at $260 \mathrm{~nm}$ 116 and $280 \mathrm{~nm}$ (Nanodrop ND1000, Thermo Scientific, Villebon sur Yvette, France). All 
117 genotyping procedures were carried out by Integragen (Evry, France). Concerning the whole

118 genome genotyping, the procedure was as follows: $200 \mathrm{ng}$ of DNA was hybridized overnight

119 to HumanOmniExpress BeadChips (Illumina, San Diego, CA, USA), allowing the analysis of

120 approximately $7.33 \times 10^{5}$ SNPs per DNA sample. Unhybridized and non-specifically

121 hybridized DNA was washed out. The BeadChips were then stained and scanned on an

122 Illumina iScan. Detailed methods are provided in the Infinium HD Assay Ultra Protocol

123 Guide from Illumina. Concerning the 40 other SNPs (see "Choice of candidate genes"), they 124 were genotyped as previously described [40].

\section{Postprandial experiments}

127 Subjects were asked to refrain from consuming LYC-rich foods (tomatoes, tomato 128 products, foods rich in tomato, watermelon) for $48 \mathrm{~h}$ before the postprandial clinic visit. In 129 addition, the day prior to the visit, they were asked to eat dinner between 7 and 8 p.m., 130 without any alcohol intake. They were also asked to abstain from consuming any food or 131 beverage other than water after the diner and until the clinic visit. After the overnight fast,

132 they arrived at the local Center for Clinical Investigation (la Conception Hospital, Marseille,

133 France) and consumed a test meal including $100 \mathrm{~g}$ of tomato puree purchased from a local 134 supermarket providing $9.7 \mathrm{mg}$ all-trans LYC as measured by high performance liquid 135 chromatography (HPLC). The puree also contained around $0.5 \mathrm{mg}$ cis isomers of LYC as 136 evaluated by the relative peak area of all the cis-isomers (4 peaks identified) as compared to 137 the peak area of the all-trans isomer in the HPLC chromatograms. This LYC dose allowed us 138 to deliver about twice the mean dietary intake of LYC in France and a LYC dose close to 139 daily intake in the United states [30, 38]. The remainder of the test meal consisted of semolina 140 (70 g) cooked in $200 \mathrm{~mL}$ of hot water, white bread (40 g), egg whites (60 g), peanut oil (50 g), 141 and mineral water $(330 \mathrm{~mL})$. The subjects were asked to consume the meal at a steady pace, 
142 with half of the meal consumed in the first $10 \mathrm{~min}$, and the remainder of the meal consumed

143 in the 20 next min. This pacing should have ultimately reduced anyvariability in gastric

144 emptying due to variation in rates of intake. No other food was permitted over the following 8

$145 \mathrm{~h}$, but subjects were permitted to finish the remainder of the $330 \mathrm{~mL}$ of water they had not

146 drunk during the meal. A fasting baseline blood sample was drawn before administration of

147 the meal as well as at 2, 3, 4, 5, 6 and $8 \mathrm{~h}$ after meal consumption. Blood was taken up into

148 evacuated tubes containing K-EDTA. The tubes were immediately placed into an ice-water

149 bath and covered with aluminum foil to avoid light exposure. Plasma was isolated by

150 centrifugation $\left(10 \mathrm{~min}\right.$ at $4{ }^{\circ} \mathrm{C}$ and $\left.878 \mathrm{~g}\right)$ within $2 \mathrm{~h}$ following collection.

152 CM preparation

153 Plasma $(6 \mathrm{~mL})$ was overlaid with $0.9 \% \mathrm{NaCl}$ solution $(4.5 \mathrm{~mL})$ and centrifuged for 28 $154 \mathrm{~min}$ at $130,000 \mathrm{x} g$ at $10{ }^{\circ} \mathrm{C}$ using a SW41Ti rotor (Beckman Coulter, Villepinte, France) in a

155 Thermo Sorvall WX100 ultracentrifuge (Thermo Scientific, Saint Herblain, France). The 156 upper phase, containing mainly $\mathrm{CM}$ and large $\mathrm{CM}$ remnants [41, 42], was collected. 157 Immediately after recovery, $\mathrm{CM}$ were stored at $-80{ }^{\circ} \mathrm{C}$ prior to $\mathrm{LYC}$ analysis.

CM LYC extraction and analysis

Total CM LYC, a mixture of all-trans and LYC cis isomers [42, 43], was extracted and

161 analyzed as follows. Briefly, up to $2 \mathrm{~mL}$ of $\mathrm{CM}$ was deproteinated by adding one volume of 162 ethanol which also contained apo-8'-carotenal as an internal standard. After adding two 163 volumes of hexane, the mixture was vortexed for $10 \mathrm{~min}$ and centrifuged at $500 \times \mathrm{x}$ for 10 $164 \min$ at $4{ }^{\circ} \mathrm{C}$. The upper phase (containing LYC) was collected and the sample was extracted a second time with hexane following the same procedure. The hexane phases were pooled and

166 evaporated completely under nitrogen gas. The dried extract was then redissolved in $200 \mu \mathrm{L}$ 
167 of a dichloromethane/methanol mixture $(65: 35, \mathrm{v} / \mathrm{v})$. All extractions were performed at room

168 temperature under yellow light to minimize light-induced damage. A volume of $90 \mu \mathrm{L}$ was

169 injected for HPLC analysis. Separation was achieved using a $10 \times 4.0 \mathrm{~mm}, 2 \mu \mathrm{m}$ Modulo-

170 Cart QS guard column (Interchim, Montluçon, France) followed by a $250 \times 4.6 \mathrm{~mm}$ internal

171 diameter, $5 \mu \mathrm{m}$ particle size, YMC C30 column (Interchim) held at $35{ }^{\circ} \mathrm{C}$. The mobile phase

172 was composed of HPLC grade methanol (A), methyl tert-butyl ether (B) and water (C). A

173 linear gradient of $96 \% \mathrm{~A}, 2 \% \mathrm{~B}, 2 \% \mathrm{C}$ at $\mathrm{t}=0$ to $18 \% \mathrm{~A}, 80 \% \mathrm{~B}, 2 \% \mathrm{C}$ at $\mathrm{t}=27 \mathrm{~min}$ at a flow

174 rate of $1 \mathrm{~mL} / \mathrm{min}$ was used. The HPLC system consisted of a pump (Waters 2690) connected

175 in-line with a photodiode-array detector (Waters 2996) (Waters, Saint Quentin en Yvelines,

176 France). LYC was identified via UV-Vis spectra and retention time coincident with authentic

177 standard (generous gift of DSM LTD, Basel, Switzerland) and quantitated at $472 \mathrm{~nm}$. The

178 consecutive peaks, which contained all-trans LYC and cis-LYC isomers, were integrated

179 together to obtain the sum of all LYC isomers. Integration was performed using Chromeleon

180 software (version 6.80, Dionex, Villebon sur Yvette, France), and quantitation was performed

181 by comparing sample peak area with all-trans LYC calibration curves. Values were corrected

182 by extraction efficiency based on the recovery of the internal standard.

183

184 Calculations

185 The trapezoidal approximation rule was used to calculate the postprandial plasma CM

186 LYC response, i.e. the area of the postprandial plasma CM LYC concentration over 8 hours

187 (AUC). Calculated AUCs were baseline-corrected using the fasting plasma CM LYC 188 concentration measured in each subject.

189

190

Choice of candidate genes 
192 vitro methods to be involved in cellular uptake of LYC, i.e. SCARB1 (scavenger receptor class

193 B, member 1) [19] and CD36 (cluster of differenciation 36) [20], genes that are suspected to

194 be involved, directly or indirectly, in enterocyte LYC metabolism, e.g. L-FABP (liver-fatty

195 acid binding protein) [24], MTTP (microsomal triglyceride transfer protein), and genes that

196 have been associated in genome-wide association studies [23, 36] or candidate gene

197 association studies [30, 33-35] with blood LYC concentration. This resulted in the selection

198 of 26 genes (Supplementary table 1), representing 2202 SNPs on the arrays. In addition, we

199 added 30 SNPs in 23 genes that we have recently found to be associated with the postprandial

200 CM triacylglycerol response in the same group of subjects [44]. Indeed, CMs are the main

201 blood carrier of newly absorbed LYC and we hypothesized that genetic variants that affect the

202 secretion/clearance of CMs in the postprandial period likely affect the postprandial blood

203 response in LYC. We also added 40 SNPs that have been associated, in previous publications,

204 with lipid metabolism (Supplementary table 2). After genotyping of the subjects (see

205 above), SNPs whose genotype call rate was $<95 \%$, or SNPs presenting a significant departure

206 from Hardy-Weinberg equilibrium $(P<0.05$ following the Chi-squared test), were excluded

207 from all subsequent analysis (387 SNPs excluded; 1885 SNPs left for the PLS regression 208 analysis).

209

210 -Multivariate analysis with partial least squares regression

211 In order to identify SNPs associated with the variability in the postprandial plasma

212 CM LYC response, we employed partial least squares regression (PLS). PLS is a multivariate

213 statistical tool often used for chemometric [45] and spectrometric modeling, and which has

214 recently been applied to SNP-based predictions by our group [44, 46] and others [47, 48]. Due

215 to the large number of SNPs compared with the low number of subjects and multicollinearity 
216 between SNPs, PLS regression is used to identify the best combination of variables (i.e.

217 SNPs) that are predictive of another variable, in this case the postprandial CM LYC response.

218 Since the inheritance model could not be known for the 1885 SNPs entered in the PLS

219 regression analysis, a general genetic model was assumed, i.e. the three genotypes of each

220 SNP were treated as separate categories with no assumption made about the effect conferred

221 by homozygous or heterozygous alleles on the phenotype. Different PLS regression models

222 were built using increasing variable in the projection (VIP) threshold values. The model

223 maximizing the explained $\left(\mathrm{R}^{2}\right)$ and the predicted variance $\left(\mathrm{Q}^{2}\right)$, and validated following cross-

224 validation ANOVA, was selected. Additional validation procedures of the PLS regression

225 models [37, 44, 49] were also performed and are described in Supplementary Methods.

226 Simca-P12 software (Umetrics, Umeå, Sweden) was used for all multivariate data analyses

227 and modeling.

228

229

Univariate analysis

230 In a second approach, we performed univariate analyses to compare the postprandial

231 plasma CM LYC response between subgroups of subjects who bore different genotypes for

232 the SNPs selected in the PLS regression model. Differences obtained in the different genotype

233 subgroups were analyzed using Student's t-test with the Benjamini-Hochberg correction,

234 using QVALUE software [50]. For all tests, an adjusted $P$-value $<0.05$ was considered

235 significant.

236 
Results

238 Interindividual variability in postprandial plasma CM LYC responses to the test meal

Figure 1 shows the postprandial CM LYC response after consumption of the test meal containing tomato puree. The CV of the postprandial plasma CM LYC response, called "LYC

241 response" hereafter in the manuscript, was 70\%, as illustrated in Figure 2.

Correlation between the LYC response to the test meal and the fasting plasma LYC concentration

In order to assess whether the fasting plasma LYC concentration, previously found to be a good marker of LYC status, was related to LYC bioavailability, we calculated the correlation between the LYC response of the subjects and their fasting plasma LYC concentration. Results showed that there was a positive relationship between these two variables (Pearson's r=0.37, $P<0.05)$.

Genetic variants associated with the LYC response

As explained in the subjects and methods section, we used PLS regression to examine whether the 1885 candidate SNPs (used as qualitative $\mathrm{X}$ variables) could explain a significant part of the variability in the LYC response of the subjects. As shown in Table 2, the model including all SNPs explained the group variance with good accuracy $\left(\mathrm{R}^{2}=0.95\right)$ but was not

256 predictive of this variance $\left(\mathrm{Q}^{2}=-0.1\right)$. Therefore, to improve the model and find an association of SNPs more predictive of the LYC response, we filtered out SNPs that made no important 258 contribution, i.e. those who displayed the lowest variable importance in the projection (VIP) values. After applying several thresholds of VIP value (Table 2), we found that the best model obtained included 44 SNPs (28 not in linkage disequilibrium, plus 16 in linkage

261 disequilibrium, shown in Supplementary table 3). The 28 SNPs were located in or near 16 
262 genes (Table 3) and explained $72 \%$ of the group variance, with a prediction index $\mathrm{Q}^{2}$ of $56 \%$

263 (Table 2). The robustness and the stability of the model were validated by three additional 264 methods detailed in the Supplementary Methods section.

265 Using univariate statistics, the association of the 28 SNPs with the LYC response was 266 further evaluated by comparing, for each SNP, the LYC response of subjects who bore 267 different genotypes (Table 3). For 11 of the 28 SNPs, subjects who bore different genotypes 268 exhibited a significantly different LYC response (q-value $<0.05)$.

269

270

Genetic score to calculate the LYC response of a genotyped subject

Knowing a subject's genotype at the 28 aforementioned loci, it is possible to calculate

272 his ability to respond to LYC, according to the following equation:

$$
\widehat{R P}=a+\sum_{1}^{28} r i * \text { genotype. }(S N P i)
$$

273 With RP as the responder phenotype (i.e. the LYC response), $a$ as a constant, $r_{i}$ as the 274 regression coefficient of the $i^{\text {th }}$ SNP included in the PLS regression model, and 275 genotype. $\left(S N P_{i}\right)$ as a Boolean variable indicating the subject's genotype at the $i^{\text {th }} \mathrm{SNP}$. A list 276 of the regression coefficients calculated by the SIMCA software can be found in 277 Supplementary table 5.

278 


\section{Discussion}

The first key observation of this study was the high interindividual variability observed

281 in the LYC response to the test meal containing tomato puree. The $70 \% \mathrm{CV}$ is in agreement

282 with previous reports [18, 26-30]. Until very recently, the reason for this high interindividual

283 variability was not known. Only one study has attempted to link this variability with SNPs in

284 two key genes of carotenoid metabolism, i.e. $B C O 1$ and $B C O 2$, by observing plasma LYC

285 response following a 3 week supplementation with tomato or watermelon juice [30]. This data

286 suggested that subjects with the AA/CT genotype combination at rs12934922 and rs7501331

287 in $B C O 1$ were more responsive to LYC than those without this genotype combination.

288 However, numerous SNPs in genes related to other aspects of lycopene absorption and

289 excretion likely impact LYC response. Indeed, we have recently shown that $73 \%$ of the

290 interindividual variability in the bioavailability of the carotenoid lutein can be explained by a

291 combination of 29 SNPs in genes involved in lutein and lipid metabolism [37]. Moreover,

292 LYC bioavailability, is likely affected by multiple parameters besides LYC absorptione.g.

293 catabolism, tissue uptake, oxidation, etc. Therefore, we assessed the involvement of SNPs in

294 all genes suspected to modulate this phenotype.

295 The result of the multivariate analysis, whose validity was verified by several tests,

296 demonstrated that a significant part $(72 \%)$ of the interindividual variability in the LYC

297 response could be explained by 28 SNPs in or near 16 genes. Seven out of these 16 genes, i.e.

298 ABCA1, LPL, INSIG2, SLC27A6, LIPC, CD36 and APOB, have recently been found to be

299 involved in the postprandial CM triacylglycerol response in the same group of subjects [44].

300 This was not surprising as most newly absorbed LYC is carried from the intestine to

301 peripheral organs via CM, which are mainly composed of triacylglycerols. Since this study

302 focuses on LYC bioavailability, we will only discuss the potential role of the 4 genes that

303 were not associated with the CM triacylglycerol response and that had SNPs which displayed 
304 a significant corrected $P$-value (Table 3). These genes were ELOVL2 (ELOVL fatty acid 305 elongase 2), MTTP (microsomal triglyceride transfer protein), ABCB1 (ATP-binding cassette, 306 sub-family B, member 1) and SOD2 (superoxide dismutase 2, mitochondrial). First, it is 307 striking to observe that 3 of the top 4 SNPs identified in the PLS model were related to 308 ELOVL2. This result suggests that this gene plays a key role in the LYC response. ELOVL2 is 309 known to catalyze the elongation of EPA (eicosapentaenoic acid) to DPA (docosapentaenoic 310 acid) and DPA to DHA (docosahexaenoic acid). LYC is not considered to be a substrate for 311 this enzyme, thus, this association is intriguing. Nevertheless, the fact that rs3798709 and 312 rs9468304 in ELOVL2 were also associated with both the lutein [37] and $\beta$-carotene response 313 (data not shown submitted to publication) strongly supports its involvement in carotenoid 314 metabolism. Three SNPs in MTTP were also found in the selected PLS model. MTTP encodes 315 for the microsomal triglyceride transfer protein, which is involved in the packaging of 316 triacylglycerols within the CM. Its association with the LYC response is therefore not 317 surprising, as LYC is incorporated into $\mathrm{CM}$ in the enterocyte. $A B C B 1$ encodes for the $\mathrm{P}$ 318 glycoprotein, an ATP-dependent drug efflux pump for xenobiotic compounds with broad 319 substrate specificity. To the best of our knowledge, there is currently no study on the 320 involvement of this protein in LYC transport, but the present association suggests that this 321 protein may participate in LYC absorption, possibly by effluxing a fraction of uptaken LYC back to the intestinal lumen. The low absorption efficiency of LYC, about $30 \%$ at the dose of $32310 \mathrm{mg}$ [18], supports this hypothesis, but further studies are required to determine ABCA1 324 involvement. Our results also suggest that SOD2, responsible for converting superoxide by325 products to hydrogen peroxide and diatomic oxygen, is involved in LYC status, possibly 326 through impacts on LYC metabolism. We hypothesize that when this enzyme is not efficient, 327 superoxide by-products are quenched by LYC, leading to its degradation. 

metabolism were absent from the selected PLS model. These genes are SCARB1 and CD36,

330 who code for proteins involved in the cellular uptake of LYC [19] [20], and BCO2 [10] and

331 BCO1 [22], which have been shown in vitro to cleave LYC. The fact that SCARB1 was not 332 associated with LYC bioavailability was surprising as this scavenger receptor has been 333 involved in cellular uptake of LYC by intestinal cells $[19,51]$ and a recent study showed that 334 a SNP in this gene was associated with blood LYC concentration [36]. The results with $B C O 1$ are in contradiction with the work by Wang et al., cited earlier [30] but match the findings for BCO2. We have three main hypotheses to explain these lacks of association. The first hypothesis is that SNPs genotyped in these genes do not result in a functionally different 338 phenotype with regards to LYC bioavailability. The second hypothesis is that the association of SNPs in these genes is weaker than that of the SNPs in the selected model. In other words, these SNPs may play a minor role in LYC bioavailability. This is not in disagreement with the

341 fact that they can play a significant role in fasting blood LYC concentrations. For example,

342 SR-BI is expressed in several tissues which could explain that genetic variants in this gene 343 play a key role in, for example, blood LYC uptake by other tissues while they do not play a 344 key role in LYC uptake by the intestine.. Finally, the third hypothesis is that some SNPs in 345 these genes were not entered in the PLS regression analysis because either they were not 346 expressed on the BeadChips, or they were excluded from the analysis (for not following the 347 Hardy-Weinberg equilibrium or because their genetic call rate was $<95 \%$ ). While we 348 acknowledge these limitations of the study, the primary results remain, i.e. a significant 349 fraction of the variability in LYC bioavailability is associated with a combination in genetic 350 variants identified in our model. After determining that LYC bioavailability is, at least partly, modulated by a 352 combination of SNPs, the obvious question that arose was : "do these SNPs impacting LYC 
353 bioavailability affect long-term blood LYC status?" To answer this question, we assessed

354 whether there was a relationship between the LYC response of the volunteers to the tomato 355 puree test meal and their fasting plasma LYC concentrations. The result of this investigation,

356 which showed a significant relationship between the two parameters, suggests that the ability 357 to respond to dietary LYC is implicated in long term blood, and likely tissue, LYC 358 concentrations. Nevertheless, the relatively low correlation coefficient, i.e. 0.37 , supports the 359 assumption that other factors, e.g. LYC catabolism, oxidative stress..., are involved in long 360 term blood LYC concentrations.

362 To conclude, the results of this study demonstrate for the first time that interindividual

363 variability in LYC bioavailability is, at least partly, genetically controlled. They also suggest 364 that ELOVL2 is implicated in LYC, and more generally, carotenoid metabolism. Furthermore, 365 the ability to respond to dietary LYC is a determinant of circulating fasting LYC 366 concentrations. These associations will need to be validated in other populations, which will 367 also allow for the identification of additional SNPs not included in our candidates based on 368 the current knowledge of LYC metabolism, which is still quite limited. The ultimate objective 369 of these studies will be to provide nutritionists with an accurate and validated genetic tool to 370 predict one's individual LYC bioavailability for optimal LYC intake. 


\section{Tables}

Table 1. Characteristics of the subjects included in the statistical analysis of the results.

Parameter $\quad$ Mean $^{\prime} \pm$ SEM

Age (years)

Weight $(\mathrm{kg})$

$\operatorname{BMI}\left(\mathrm{kg} / \mathrm{m}^{2}\right)$

Glucose $(\mathrm{m} M)^{2}$

Triacylglycerol $(\mathrm{g} / \mathrm{L})^{2}$

Total cholesterol $(\mathrm{g} / \mathrm{L})^{2}$

Hemoglobin $(\mathrm{g} / \mathrm{dL})^{2}$

$\operatorname{LYC}(\mu M)^{2,3}$
$32.6 \pm 2.1$

$72.6 \pm 1.3$

$22.8 \pm 0.3$

$4.7 \pm 0.1$

$0.7 \pm 0.1$

$1.7 \pm 0.1$

$15.0 \pm 0.1$

$2.3 \pm 0.3$

${ }^{1} \mathrm{n}=33$.

${ }^{2}$ Fasting plasma variables.

LYC, lycopene. 
Table 2. Performances of different partial least squares regression models to explain the postprandial chylomicron lycopene response. ${ }^{1}$

\begin{tabular}{|c|c|c|c|c|}
\hline VIP threshold & $R^{2}$ & $Q^{2}$ & SNPs no. & $P(C V-A N O V A)$ \\
\hline No selection & 0.94 & -0.10 & 1885 & 1 \\
\hline$>0.5$ & 0.87 & -0.10 & 604 & 1 \\
\hline$>1.0$ & 0.70 & 0.01 & 183 & 0.808 \\
\hline$>1.5$ & 0.73 & 0.57 & 61 & 0.109 \\
\hline$>1.55$ & 0.72 & 0.55 & 58 & 0.104 \\
\hline$>1.6$ & 0.71 & 0.52 & 56 & 0.104 \\
\hline$>1.65$ & 0.71 & 0.55 & 50 & 0.072 \\
\hline$>1.7$ & 0.72 & 0.55 & 45 & 0.041 \\
\hline$>1.75$ & 0.72 & 0.56 & 44 & 0.037 \\
\hline$>1.8$ & 0.70 & 0.45 & 32 & 0.047 \\
\hline$>1.85$ & 0.66 & 0.37 & 25 & 0.025 \\
\hline$>1.9$ & 0.63 & 0.36 & 23 & 0.037 \\
\hline$>1.95$ & 0.60 & 0.34 & 20 & 0.040 \\
\hline$>2.0$ & 0.62 & 0.39 & 16 & 0.036 \\
\hline$>2.1$ & 0.62 & 0.39 & 15 & 0.029 \\
\hline
\end{tabular}

${ }^{1}$ Different PLS regression models were built using increasing VIP threshold values. The model maximizing the explained $\left(\mathrm{R}^{2}\right)$ and the predicted variance $\left(\mathrm{Q}^{2}\right)$, and validated following cross-validation ANOVA plus three other validation methods (described in Supplementary Material), was selected. This is the model with VIP $>1.75$, shown in the table.

CV-ANOVA, cross-validation ANOVA; $\mathrm{Q}^{2}$, predicted variance; $\mathrm{R}^{2}$, explained variance; SNP, single nucleotide polymorphism; VIP, variable importance in the projection. 
Table 3. Genes and single nucleotide polymorphisms associated with the postprandial chylomicron lycopene response. ${ }^{1}$

\begin{tabular}{|c|c|c|c|}
\hline Gene and SNP rs no. ${ }^{2}$ & VIP value & SNP minor allele frequency & $\begin{array}{l}\text { Adjusted } \\
P \text {-value }\end{array}$ \\
\hline ELOVL2-rs3798709 & 3.38 & 0.252 & 0.030 \\
\hline ELOVL2-rs9468304 & 3.33 & 0.302 & 0.030 \\
\hline$L P L$-rs 7005359 & 3.19 & 0.298 & 0.030 \\
\hline ELOVL2-rs911196 & 3.07 & 0.252 & 0.030 \\
\hline$A B C A 1-\mathrm{rs} 4149316$ & 2.81 & 0.122 & 0.023 \\
\hline MTTP-rs17029173 & 2.73 & 0.135 & 0.030 \\
\hline$A B C B 1$-rs 10248420 & 2.67 & 0.347 & 0.030 \\
\hline PKD1L2-rs935933 & 2.57 & 0.201 & 0.053 \\
\hline$M T T P$-rs 1032355 & 2.56 & 0.251 & 0.067 \\
\hline$A B C G 2$-rs 1871744 & 2.55 & 0.173 & 0.061 \\
\hline$A B C A 1-\mathrm{rs} 2791952$ & 2.50 & 0.140 & 0.030 \\
\hline$L P L$-rs 7841189 & 2.44 & 0.148 & 0.063 \\
\hline INSIG2-rs17006621 & 2.27 & 0.172 & 0.077 \\
\hline$A B C A 1-r s 3887137$ & 2.15 & 0.123 & 0.076 \\
\hline MTTP-rs745075 & 2.03 & 0.061 & 0.030 \\
\hline NPC1L1-rs17725246 & 1.97 & 0.254 & 0.106 \\
\hline$L P L-\mathrm{rs} 17482753$ & 1.95 & 0.088 & 0.101 \\
\hline SOD2-rs9365046 & 1.93 & 0.169 & 0.039 \\
\hline SLC27A6-rs 10053477 & 1.91 & 0.209 & 0.090 \\
\hline$A B C A 1-\mathrm{rs} 1331924$ & 1.88 & 0.245 & 0.091 \\
\hline$I S X$-rs2056983 & 1.85 & 0.117 & 0.092 \\
\hline
\end{tabular}




\begin{tabular}{lccc}
\hline$L I P C$-rs12914035 & 1.85 & 0.096 & 0.146 \\
$C D 36$-rs4112274 & 1.85 & 0.224 & 0.030 \\
$P N L I P$-rs11197742 & 1.84 & 0.087 & 0.086 \\
$A B C A 1$-rs4149299 & 1.84 & 0.082 & 0.119 \\
$A P O B$-rs1042031 & 1.76 & 0.128 & 0.157 \\
$L I P C$-rs8035357 & 1.76 & 0.150 & 0.101 \\
$A B C B 1$-rs10280101 & 1.75 & 0.145 & 0.101 \\
\hline
\end{tabular}

${ }^{1}$ SNPs present in the selected PLS regression model shown in Table 2.

${ }^{2}$ SNPs are ranked by decreasing VIP value. Note that 16 out of the 44 SNPs present in the selected model were in linkage disequilibrium. Since these SNPs provided redundant information, we randomly kept one of each SNP (those presented in this table) in the final selected PLS model. The SNPs in linkage disequilibrium with some of these SNPs are shown in Supplemental table 2.

${ }^{3}$ Student's t-test with the Benjamini-Hochberg correction was carried out to determine differences between the postprandial CM LYC response according to genotype groups for each SNP.

SNP, single nucleotide polymorphism; rs, reference SNP; VIP, variable importance in the projection. A complete list of gene names and symbols can be found in Supplementary table 1. 


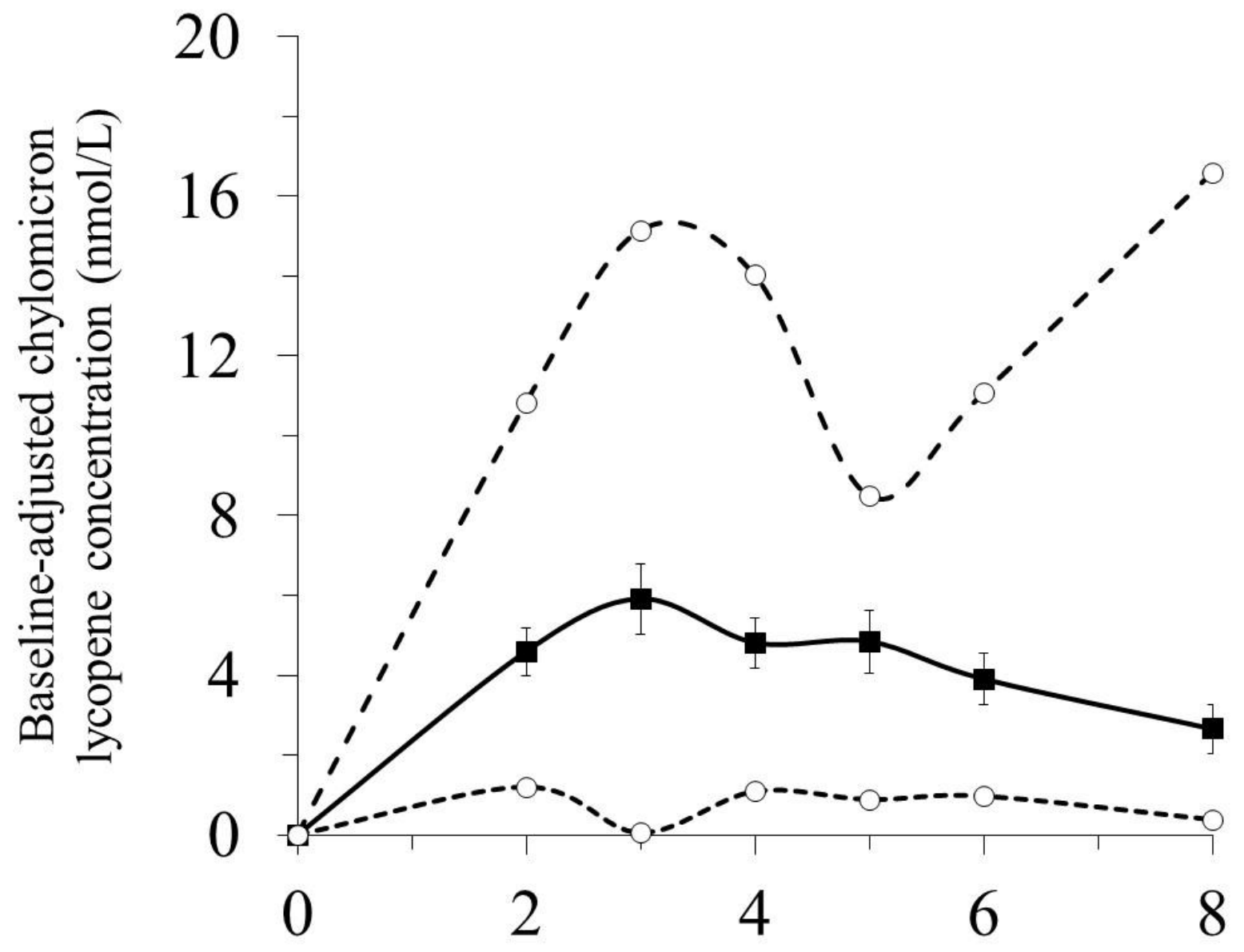

\section{Figures}

Figure 1: Baseline-adjusted chylomicron lycopene (CM LYC) concentration over 8 h after consumption of the test meal containing tomato puree.

For each subject, postprandial CM LYC concentrations were baseline-adjusted by normalizing to the fasting CM LYC concentration. The bold curve shows mean \pm SEM of 33 subjects combined. The smaller dashed curve shows the concentration of CM LYC measured in the lowest responder. The larger dashed curve shows the concentration of CM LYC measured in the highest responder. 


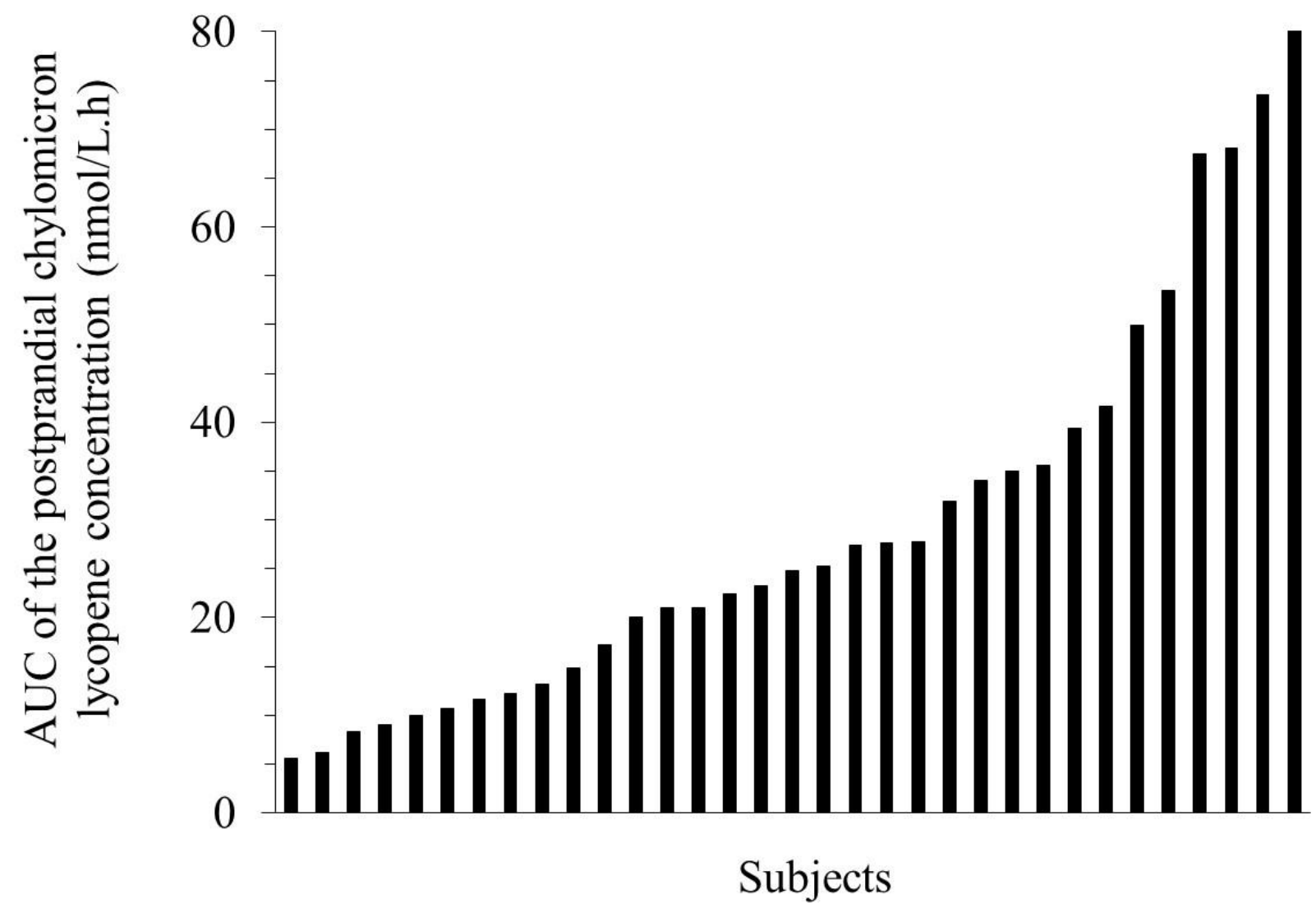

Figure 2: Individual AUCs of the postprandial CM LYC response after consumption of the test meal containing tomato puree.

Subjects were sorted by increasing postprandial CM LYC response (i.e., 0-8 h AUC). 


\section{Acknowledgements}

We are very grateful to Dr. Rachel Kopec for critical comments on the manuscript.

The authors have no conflict of interest to declare.

The present work has received research funding from the European Community's Sixth Framework Programme. The funding was attributed to the Lycocard project $\left(\mathrm{n}^{\circ} 016213\right)$ which was an Integrated Project within the framework of the "Food Quality and Safety" programme. This publication reflects only the view of the authors. The Lycocard community is not liable for any use that may be made of the results.

\section{Authors' contributions to manuscript:}

PB designed research; MN conducted the clinical research; RB analysed lycopene by HPLC;

CD and PB analyzed data and CD performed statistical analysis; $\mathrm{PB}$ and CD wrote the paper; PB and CD had primary responsibility for final content. 


\section{References}

[1] Story, E. N.; Kopec, R. E.; Schwartz, S. J.; Harris, G. K. An update on the health effects of tomato lycopene. Annu. Rev. Food Sci. Technol. 1:189-210; 2010.

[2] Mordente, A.; Guantario, B.; Meucci, E.; Silvestrini, A.; Lombardi, E.; Martorana, G. E.; Giardina, B.; Bohm, V. Lycopene and cardiovascular diseases: an update. Curr. Med. Chem. 18:1146-1163; 2011.

[3] Bohm, V. Lycopene and heart health. Mol. Nutr. Food Res. 56:296-303; 2012.

[4] Giovannucci, E. Tomatoes, tomato-based products, lycopene, and cancer: Review of the epidemiologic literature. J. Natl. Cancer Inst. 91:317-331; 1999.

[5] Viuda-Martos, M.; Sanchez-Zapata, E.; Sayas-Barbera, E.; Sendra, E.; Perez-Alvarez, J. A.; Fernandez-Lopez, J. Tomato and tomato byproducts. Human health benefits of lycopene and its application to meat products: a review. Crit. Rev. Food Sci. Nutr. 54:1032-1049; 2014.

[6] Biddle, M. J.; Lennie, T. A.; Bricker, G. V.; Kopec, R. E.; Schwartz, S. J.; Moser, D. K. Lycopene Dietary Intervention: A Pilot Study in Patients With Heart Failure. $J$. Cardiovasc. Nurs.; 2014.

[7] Kelkel, M.; Schumacher, M.; Dicato, M.; Diederich, M. Antioxidant and antiproliferative properties of lycopene. Free Radic. Res. 45:925-940; 2011.

[8] Kopec, R. E.; Riedl, K. M.; Harrison, E. H.; Curley, R. W., Jr.; Hruszkewycz, D. P.; Clinton, S. K.; Schwartz, S. J. Identification and quantification of apo-lycopenals in fruits, vegetables, and human plasma. J. Agric. Food Chem. 58:3290-3296; 2010.

[9] Erdman, J. W., Jr.; Ford, N. A.; Lindshield, B. L. Are the health attributes of lycopene related to its antioxidant function? Arch. Biochem. Biophys. 483:229-235; 2009. 
[10] Lindshield, B. L.; Canene-Adams, K.; Erdman, J. W., Jr. Lycopenoids: are lycopene metabolites bioactive? Arch. Biochem. Biophys. 458:136-140; 2007.

[11] Mein, J. R.; Lian, F.; Wang, X. D. Biological activity of lycopene metabolites: implications for cancer prevention. Nutr. Rev. 66:667-683; 2008.

[12] Gouranton, E.; Thabuis, C.; Riollet, C.; Malezet-Desmoulins, C.; El Yazidi, C.; Amiot, M. J.; Borel, P.; Landrier, J. F. Lycopene inhibits proinflammatory cytokine and chemokine expression in adipose tissue. J. Nutr. Biochem. 22:642-648; 2011.

[13] Aydemir, G.; Kasiri, Y.; Birta, E.; Beke, G.; Garcia, A. L.; Bartok, E. M.; Ruhl, R. Lycopene-derived bioactive retinoic acid receptors/retinoid-X receptors-activating metabolites may be relevant for lycopene's anti-cancer potential. Mol. Nutr. Food Res. 57:739-747; 2013.

[14] Gouranton, E.; Aydemir, G.; Reynaud, E.; Marcotorchino, J.; Malezet, C.; Caris-Veyrat, C.; Blomhoff, R.; Landrier, J. F.; Ruhl, R. Apo-10'-lycopenoic acid impacts adipose tissue biology via the retinoic acid receptors. Biochim. Biophys. Acta 1811:1105-1114; 2012.

[15] Wang, X. D. Lycopene metabolism and its biological significance. Am. J. Clin. Nutr. 96:1214S-1222S; 2012.

[16] Yeum, K. J.; Russell, R. M. Carotenoid bioavailability and bioconversion. Annu. Rev. Nutr. 22:483-504; 2002.

[17] Borel, P. Factors affecting intestinal absorption of highly lipophilic food microconstituents (fat-soluble vitamins, carotenoids and phytosterols). Clin. Chem. Lab. Med. 41:979-994; 2003.

[18] Diwadkar-Navsariwala, V.; Novotny, J. A.; Gustin, D. M.; Sosman, J. A.; Rodvold, K. A.; Crowell, J. A.; Stacewicz-Sapuntzakis, M.; Bowen, P. E. A physiological 
pharmacokinetic model describing the disposition of lycopene in healthy men. J. Lipid Res. 44:1927-1939; 2003.

[19] Moussa, M.; Landrier, J. F.; Reboul, E.; Ghiringhelli, O.; Comera, C.; Collet, X.; Frohlich, K.; Bohm, V.; Borel, P. Lycopene absorption in human intestinal cells and in mice involves scavenger receptor class B type I but not Niemann-Pick C1-like 1. $J$. Nutr. 138:1432-1436; 2008.

[20] Moussa, M.; Gouranton, E.; Gleize, B.; Yazidi, C. E.; Niot, I.; Besnard, P.; Borel, P.; Landrier, J. F. CD36 is involved in lycopene and lutein uptake by adipocytes and adipose tissue cultures. Mol. Nutr. Food Res. 55:578-584; 2011.

[21] dela Sena, C.; Riedl, K. M.; Narayanasamy, S.; Curley, R. W., Jr.; Schwartz, S. J.; Harrison, E. H. The human enzyme that converts dietary provitamin A carotenoids to vitamin A is a dioxygenase. J. Biol. Chem. 289:13661-13666; 2014.

[22] dela Sena, C.; Narayanasamy, S.; Riedl, K. M.; Curley, R. W., Jr.; Schwartz, S. J.; Harrison, E. H. Substrate specificity of purified recombinant human beta-carotene 15,15'-oxygenase (BCO1). J. Biol. Chem. 288:37094-37103; 2013.

[23] Ferrucci, L.; Perry, J. R.; Matteini, A.; Perola, M.; Tanaka, T.; Silander, K.; Rice, N.; Melzer, D.; Murray, A.; Cluett, C.; Fried, L. P.; Albanes, D.; Corsi, A. M.; Cherubini, A.; Guralnik, J.; Bandinelli, S.; Singleton, A.; Virtamo, J.; Walston, J.; Semba, R. D.; Frayling, T. M. Common Variation in the beta-Carotene 15,15'-Monooxygenase 1 Gene Affects Circulating Levels of Carotenoids: A Genome-Wide Association Study. Am. J. Hum. Genet. 84:123-133; 2009.

[24] Reboul, E.; Borel, P. Proteins involved in uptake, intracellular transport and basolateral secretion of fat-soluble vitamins and carotenoids by mammalian enterocytes. Prog. Lipid Res. 50:388-402; 2011. 
[25] Dallinga-Thie, G. M.; Franssen, R.; Mooij, H. L.; Visser, M. E.; Hassing, H. C.; Peelman, F.; Kastelein, J. J.; Peterfy, M.; Nieuwdorp, M. The metabolism of triglyceride-rich lipoproteins revisited: new players, new insight. Atherosclerosis 211:1$8 ; 2013$.

[26] Stahl, W.; Sies, H. Uptake of lycopene and its geometrical isomers is greater from heatprocessed than from unprocessed tomato juice in humans. J. Nutr. 121:2161-2166; 1992.

[27] Bowen, P.; Garg, V.; Stacewiczsapuntzakis, M.; Yelton, L.; Schreiner, R. S. Variability of Serum Carotenoids in Reponse to Controlled Diets Containing Six Servings of Fruits and Vegetables per day. Ann. N Y Acad. Sci. 691:241-243; 1993.

[28] Johnson, E. J.; Qin, J.; Krinsky, N. I.; Russell, R. M. Ingestion by men of a combined dose of beta-carotene and lycopene does not affect the absorption of beta-carotene but improves that of lycopene. J. Nutr. 127:1833-1837; 1997.

[29] O'Neill, M. E.; Thurnham, D. I. Intestinal absorption of $\beta$-carotene, lycopene and lutein in men and women following a standard meal: response curves in the triacylglycerolrich lipoprotein fraction. Br. J. Nutr. 79:149-159; 1998.

[30] Wang, T. T.; Edwards, A. J.; Clevidence, B. A. Strong and weak plasma response to dietary carotenoids identified by cluster analysis and linked to beta-carotene $15,15^{\prime}-$ monooxygenase 1 single nucleotide polymorphisms. J. Nutr. Biochem. 24:1538-1546; 2013.

[31] Borel, P. Genetic variations involved in interindividual variability in carotenoid status. Mol. Nutr. Food Res. 56:228-240; 2012.

[32] Moran, N. E.; Erdman, J. W., Jr.; Clinton, S. K. Complex interactions between dietary and genetic factors impact lycopene metabolism and distribution. Arch. Biochem. Biophys. 539:171-180; 2013. 
[33] Ortega, H.; Castilla, P.; Gomez-Coronado, D.; Garces, C.; Benavente, M.; RodriguezArtalejo, F.; de Oya, M.; Lasuncion, M. A. Influence of apolipoprotein E genotype on fat-soluble plasma antioxidants in Spanish children. Am. J. Clin. Nutr. 81:624-632; 2005.

[34] Borel, P.; Moussa, M.; Reboul, E.; Lyan, B.; Defoort, C.; Vincent-Baudry, S.; Maillot, M.; Gastaldi, M.; Darmon, M.; Portugal, H.; Planells, R.; Lairon, D. Human plasma levels of vitamin E and carotenoids are associated with genetic polymorphisms in genes involved in lipid metabolism. J. Nutr. 137:2653-2659; 2007.

[35] Borel, P.; Moussa, M.; Reboul, E.; Lyan, B.; Defoort, C.; Vincent-Baudry, S.; Maillot, M.; Gastaldi, M.; Darmon, M.; Portugal, H.; Lairon, D.; Planells, R. Human fasting plasma concentrations of vitamin $\mathrm{E}$ and carotenoids, and their association with genetic variants in apo C-III, cholesteryl ester transfer protein, hepatic lipase, intestinal fatty acid binding protein and microsomal triacylglycerol transfer protein. Br. J. Nutr. 101:680-687; 2009.

[36] Zubair, N.; Kooperberg, C.; Liu, J.; Di, C.; Peters, U.; Neuhouser, M. L. Genetic variation predicts serum lycopene concentrations in a multiethnic population of postmenopausal women. J. Nutr. 145:187-192; 2015.

[37] Borel, P.; Desmarchelier, C.; Nowicki, M.; Bott, R.; Morange, S.; Lesavre, N. Interindividual variability of lutein bioavailability in healthy men: characterization, genetic variants involved, and relation with fasting plasma lutein concentration. Am. J. Clin. Nutr. 100:168-175; 2014.

[38] Porrini, M.; Riso, P. What are typical lycopene intakes? J. Nutr. 135:2042S-2045S; 2005.

[39] Hansen, T. V.; Simonsen, M. K.; Nielsen, F. C.; Hundrup, Y. A. Collection of blood, saliva, and buccal cell samples in a pilot study on the Danish nurse cohort: comparison 
of the response rate and quality of genomic DNA. Cancer Epidemiol. Biomarkers Prev. 16:2072-2076; 2007.

[40] Lecompte, S.; Szabo de Edelenyi, F.; Goumidi, L.; Maiani, G.; Moschonis, G.; Widhalm, K.; Molnár, D.; Kafatos, A.; Spinneker, A.; Breidenassel, C.; Dallongeville, J.; Meirhaeghe, A.; Borel, P. Polymorphisms in the CD36/FAT gene are associated with plasma vitamin E levels in humans. Am. J. Clin. Nutr. 93:1-8; 2011.

[41] Luchoomun, J.; Hussain, M. M. Assembly and secretion of chylomicrons by differentiated Caco-2 cells. Nascent triglycerides and preformed phospholipids are preferentially used for lipoprotein assembly. J. Biol. Chem. 274:19565-19572; 1999.

[42] Richelle, M.; Lambelet, P.; Rytz, A.; Tavazzi, I.; Mermoud, A. F.; Juhel, C.; Borel, P.; Bortlik, K. The proportion of lycopene isomers in human plasma is modulated by lycopene isomer profile in the meal but not by lycopene preparation. Br. J. Nutr. 107:1482-1488; 2012.

[43] Richelle, M.; Sanchez, B.; Tavazzi, I.; Lambelet, P.; Bortlik, K.; Williamson, G. Lycopene isomerisation takes place within enterocytes during absorption in human subjects. Br. J. Nutr. 103:1800-1807; 2010.

[44] Desmarchelier, C.; Martin, J. C.; Planells, R.; Gastaldi, M.; Nowicki, M.; Goncalves, A.; Valero, R.; Lairon, D.; Borel, P. The postprandial chylomicron triacylglycerol response to dietary fat in healthy male adults is significantly explained by a combination of single nucleotide polymorphisms in genes involved in triacylglycerol metabolism. $J$. Clin. Endocrinol. Metab. 99:E484-488; 2014.

[45] Eriksson, L.; Antti, H.; Gottfries, J.; Holmes, E.; Johansson, E.; Lindgren, F.; Long, I.; Lundstedt, T.; Trygg, J.; Wold, S. Using chemometrics for navigating in the large data sets of genomics, proteomics, and metabonomics (gpm). Anal. Bioanal. Chem. 380:419429; 2004. 
[46] Borel, P.; de Edelenyi, F. S.; Vincent-Baudry, S.; Malezet-Desmoulin, C.; Margotat, A.; Lyan, B.; Gorrand, J. M.; Meunier, N.; Drouault-Holowacz, S.; Bieuvelet, S. Genetic variants in BCMO1 and CD36 are associated with plasma lutein concentrations and macular pigment optical density in humans. Ann. Med. 43:47-59; 2010.

[47] Le Cao, K. A.; Boitard, S.; Besse, P. Sparse PLS discriminant analysis: biologically relevant feature selection and graphical displays for multiclass problems. $B M C$ Bioinformatics 12:253; 2011.

[48] Long, N.; Gianola, D.; Rosa, G. J.; Weigel, K. A. Dimension reduction and variable selection for genomic selection: application to predicting milk yield in Holsteins. $J$. Anim. Breed Genet. 128:247-257; 2011.

[49] Borel, P.; Desmarchelier, C.; Nowicki, M.; Bott, R.; Tourniaire, F. Can genetic variability in alpha-tocopherol bioavailability explain the heterogeneous response to alpha-tocopherol supplements? Antioxid. Redox. Signal.:Nov 12. [Epub ahead of print]; 2014.

[50] Storey, J. D. A direct approach to false discovery rates. Journal of the Royal Statistical Society: Series B (Statistical Methodology) 64:479-498; 2002.

[51] During, A.; Dawson, H. D.; Harrison, E. H. Carotenoid transport is decreased and expression of the lipid transporters SR-BI, NPC1L1, and ABCA1 is downregulated in Caco-2 cells treated with ezetimibe. J. Nutr. 135:2305-2312; 2005. 